\title{
Development of an Unsteady Multi-Species Numerical Method for Internal Compressible Flows in the Turbine
}

\author{
Zhong-Nan Wang, Zhi-Rong Lin and Xin Yuan \\ Key Laboratory for Thermal Science and Power Engineering of Ministry of Education, \\ Tsinghua University, Beijing 100084, P.R. China
}

\begin{abstract}
Flexible Fuel is used for land-based power generation gas turbine to meet the environmental requirements, which brings high temperature gases with variable composition to the turbine downstream.

In this paper, a high-order and high-resolution in-house CFD code (named START) was developed to capture more features of the real multi-species flow in the turbine. The code was validated with four samples, ranging from subsonic to transonic and supersonic flows. The validation showed that the code had high accuracy and fidelity to simulate the flows, which included both shock wave and species diffusion.

Then the solver was used to simulate the unsteady multi-species flow in a 1.5-stage turbine, which included $\mathrm{CO}_{2}$ coolant injection at the $1^{\text {st }}$ stator trailing edge. The cooling effect on the rotor downstream and associated losses were studied in terms of the coolant-to-free stream velocity ratio.

\section{NOMENCLATURE}

$\rho_{k} \quad=\quad$ partial density of species $k$

$Y_{k}=$ mass fraction of species $k$

$w_{j}=$ the $x_{j}$ relative velocity in the rotational coordinate

$\Omega=$ the rotation speed of the coordinate

$q_{j}=$ the thermal conduction in $x_{j}$ direction

$J_{k, j}=\quad$ the $x_{j}$ direction mass diffusion flux of species $k$

$\dot{\varpi}_{k}=$ the generation or destruction term of the species $k$

$\vartheta_{1(2)}=$ the turbulence quantities of two equation turbulence model

$D_{k}=$ the species $\mathrm{k}$ diffusion coefficient

$h_{k}=\quad$ the enthalpy of the species $k$

$V_{k, j}=\quad$ the mass diffusion velocity of species $k$ in $x_{j}$ direction
\end{abstract}

Presented at International Gas Turbine Congress 2011 Osaka, November 13-18, Osaka, Japan, IGTC2011-0125

Review completed on December 2, 2013

$$
\begin{array}{lll}
p_{k}= & \text { the partial pressure of the species } k \\
R= & \text { the gas constant, } 8.314(\mathrm{~kJ} / \mathrm{mol}) \\
M_{k}= & \text { the molecular weight of the species } k \\
V_{i}^{c}= & \text { the correction velocity in } x_{j} \text { direction } \\
\gamma & =\quad \text { the specific heat ratio of the gas mixture } \\
Y & = & \text { the stagnation pressure loss coefficient }
\end{array}
$$

\section{INTRODUCTION}

As the climate change and air pollution become a greater concern, the urgent search for a cleaner and more efficient way to convert the fossil fuel into electricity is in great need. Hydrogen or coal-derivative syngas turbines promise increased efficiency with low NOx emissions compared to natural gas based turbines[1-2]. However, the flexible fuel will exhaust hot gas with different species composition after combustion, as listed in Table 1. Hot-gases with different constituents that enter the turbine will lead to great differences in molecular weights, specific heats, and transport properties such as thermal conductivity and viscosity. These differences will eventually cause special aero-thermal behavior in the turbine compared to the conventional natural gas based turbine[3]. While the experiment method seems impossible at this high temperature, the high-fidelity multi-species numerical method should be developed to study the real high temperature multi-species flow physics in the new-generation gas turbine.

Table 1 Hot Gas Composition of Different Fuels[4]

\begin{tabular}{|c|l|l|l|l|l|}
\hline mass fraction(\%) & $\mathrm{CO} 2$ & $\mathrm{H} 2 \mathrm{O}$ & $\mathrm{N} 2$ & $\mathrm{Ar}$ & $\mathrm{O} 2$ \\
\hline Natural Gas & 7.64 & 6.11 & 77.81 & 0 & 8.44 \\
\hline Syngas & 12.23 & 4.57 & 74.01 & 0.96 & 8.23 \\
\hline Hydrogen Gas & 1.97 & 9.96 & 78.52 & 1.15 & 8.39 \\
\hline Oxy-fuel Gas & 31.25 & 65.52 & 1.66 & 1.42 & 0.14 \\
\hline
\end{tabular}

The flow in these turbines is not only multi-species but also at high speed, which is different from the flow condition in the combustion chamber. The multi-species flow in the turbine must be treated as compressible flows which may be transonic or supersonic. The discontinuity, such as shock wave, may also exit in the turbine blade passage. The hyperbolic of the flow equation becomes more significant at high speed and the acoustic wave plays a large part in the flow[5]. Each gas species is more coupled to other flow va- 
riables, such as pressure, temperature and velocity. Small error in one species will immediately be sensed by other flow components[6]. Therefore, the high-speed multi-species flow in the turbine proposes a severe challenge for numerical methods which are different from those used in the conventional combustion flow simulation.

In this paper, a high-order and high-resolution numerical method was developed for the high-speed multi-species flows in the turbine. The equations for all the species were solved in the coupled way and some modifications were made to enhance the mass conservation for each species. The in-house code was validated by four typical cases, ranging from subsonic to transonic and supersonic multi-species non-reacting flows. Finally, the multi-species flow in a 1.5-stage turbine was analyzed by the code to study the $1^{\text {st }}$ stator trailing edge coolant ejection effect on the cooling effectiveness and aerodynamic losses.

\section{PHYSICAL MODEL AND NUMERICAL METHOD}

\section{Physical Model}

The multi-species flows were governed by the extended Navier -Stokes equations, including $\mathrm{N}$ species conservation equations instead of the mass conservation equation, where $\mathrm{N}$ denotes the total number of gas species. Taken the ensemble averaged, the turbulent multi-species flow governing equation was written in tensor notation and casted in the curvilinear rotational coordinate system:

$$
\frac{\partial Q}{\partial t}+\frac{\partial F_{i}}{\partial \xi_{i}}+\frac{\partial D_{i}}{\partial \xi_{i}}+S=0
$$

where

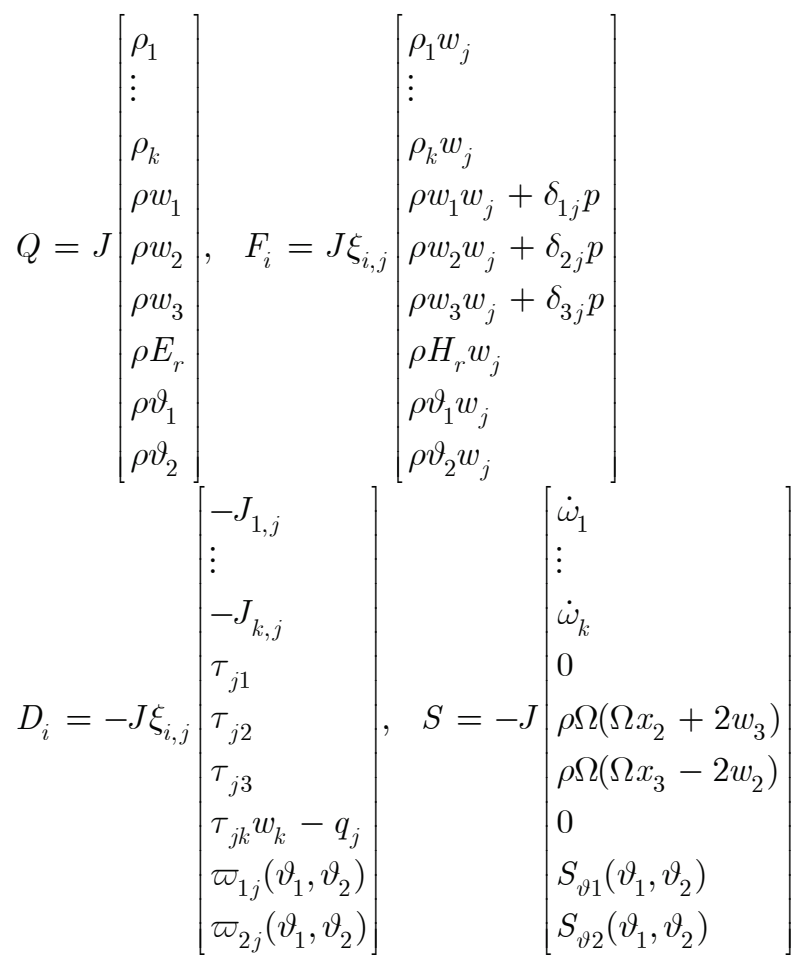

Due to the rotating effect, the centrifugal and coriolis force term was added to the source term of the momentum equation. The species mass diffusion term, shear stress and thermal conduction term were expressed as:

$$
J_{k, j}=-\rho V_{k, j}=-\rho\left(D_{k}+D_{k, t}\right) \frac{\partial Y_{k}}{\partial x_{j}}
$$

$$
\begin{gathered}
\tau_{i j}=\left(\mu+\mu_{t}\right)\left[\left(\frac{\partial w_{i}}{\partial x_{j}}+\frac{\partial w_{j}}{\partial x_{i}}\right)-\frac{2}{3} \delta_{i j} \frac{\partial w_{k}}{\partial x_{k}}\right]-\frac{2}{3} \delta_{i j} k \\
q_{j}=-\left(\kappa+\kappa_{t}\right) \frac{\partial T}{\partial x_{j}}+J_{k, j} h_{k}
\end{gathered}
$$

where

$$
Y_{k}=\frac{\rho_{k}}{\rho}
$$

The key feature of the extended Navier-Stokes equation was the species mass diffusion term, which exits in both the species and energy conservation equations. If the extended equation for multi-species flows satisfies the continuity equation, the species mass diffusion velocity should meet the following requirement:

$$
\sum_{k=1}^{N} V_{k, j}=0
$$

The implementation of the requirement will be discussed in detail at the numerical scheme section.

As to the equation of state, each species was assumed to be ideal gas and the Dalton Law was applied to obtain the mixture pressure:

$$
p=\sum_{k=1}^{N} p_{k}=\sum_{k=1}^{N} \rho_{k} \frac{R}{M_{k}} T=\rho\left(\sum_{k=1}^{N} Y_{k} R_{k}\right) T \text { (7) }
$$

Due to the high temperature in the turbine, the heat capacity for individual species was assumed to be a function of temperature[7],

$$
\begin{gathered}
C p_{k} / R_{k}=a_{1} T^{-2}+a_{2} T^{-1}+a_{3} \\
\quad+a_{4} T+a_{5} T^{2}+a_{6} T^{3}+a_{7} T^{4}
\end{gathered}
$$

The enthalpy of each species could be obtained by integration over the temperature,

$$
h=\sum_{k=1}^{N} Y_{k} h_{k}=\sum_{k=1}^{N} \int_{T_{0}}^{T} Y_{k} C p_{k}(T) d T+Y_{k} h_{k, 0}
$$

For the gas transport properties, each species' viscosity, conductivity and binary mass diffusivity were computed according to the kinetic theory. The mixture's viscosity, conductivity and mass diffusivity were obtained according to the literature[6,8-9].

\section{Numerical Method}

\section{Mass Conservation}

Mass conservation was a special issue for multi-species flows. The summation of the first $\mathrm{N}$ species equations of (1) was equivalent to the mass conservation equation in analytical form. The equivalence needed three restrictions to meet, one was the unity condition:

$$
\sum_{k=1}^{N} Y_{k}=1
$$

Another was on the diffusion velocity, that was equation (6). The third one was on the species source terms, that was

$$
\sum_{k=1}^{N} \dot{\varpi}_{s}=0
$$

In order to save computation time, the Fick's law in (2) was used to get the species diffusion. The Fick's law was only an approximation of the complex multi-species diffusion velocity system [10]. Therefore, the second restriction (6) was violated, which deteriorated the equivalence between the species conservation equation and continuity equation. So the system was over-determined with $\mathrm{N}$ independent unknowns and $\mathrm{N}+1$ equations.

In general, there were two methods to fix this problem. The first and straight-forward method was to solve the global continuity equation and $\mathrm{N}-1$ species conservation equations. The last species mass fraction was obtained by using the unity condition (10) and absorbed all the inconsistencies introduced by (2). It was dangerous when the mass fraction was comparable quantitatively and all 
variables were strongly coupled at high speed. The error would propagate immediately to other variables.

To overcome this difficulty, the second method seemed more reasonable, which was implemented in our in-house code. The equation (1) was treated in a coupled way, which $\mathrm{N}$ species conservation equations were solved simultaneously instead of continuity equation and $\mathrm{N}-1$ species equations. In order to satisfy the global mass conservation, the correction velocity was introduced to modify the inaccurate diffusion velocity

$$
\frac{\partial \rho Y_{k}}{\partial t}+\frac{\partial \rho Y_{k}\left(w_{i}+V_{i}^{c}\right)}{\partial x_{i}}=\frac{\partial}{\partial x_{i}}\left(\rho Y_{k} V_{k, i}\right)+\dot{\omega}_{k}
$$

where the correction velocity was calculated by

$$
V_{i}^{c}=\sum_{k=1}^{N} V_{k, i}
$$

Then the summation of the equations (12) over species number $\mathrm{N}$ will fall into the global mass conservation equation. This coupled method caught the essence of the inconsistence. It conserved the mass from both the individual species' and the global mixture's view.

\section{Pressure Oscillation}

For multi-species flows, the specific heat ratio was a function of field. It followed the transport equation

$$
\frac{\partial}{\partial t}\left(\frac{1}{\gamma-1}\right)+w_{i} \frac{\partial}{\partial x_{i}}\left(\frac{1}{\gamma-1}\right)=0
$$

which could be derived from the energy equation.

At the material interface, the requirement (14) was violated by calculating specific heat ratio

$$
\gamma=\sum_{k=1}^{N} Y_{k} C p_{k} / \sum_{k=1}^{N} Y_{k} C v_{k}
$$

When we calculate the pressure from the internal energy

$$
p=(\gamma-1) \rho e
$$

the error will accumulate on the pressure, contributing to the pressure oscillation near the material interface. This problem was more obvious in the high-speed multi-species flow.

In our code, the additional equation (14) was solved to avoid the pressure oscillation at the material interface.

\section{Numerical Scheme}

The full-species equations (1) was solved in the coupled way implicitly using the LU-SGS-GE time marching scheme[11], which had high robustness and convergence rate. The spatial derivative was discretized by a fourth-order MUSCL TVD scheme[12], which had high resolution to capture the flow features in detail.

\section{VALIDATION SAMPLES}

The validation was carried out step by step to testify to the code's accuracy and fidelity to simulate the high-speed multi-species flow. The validation samples were organized extensively and systematically, in which the speed ranged from subsonic, transonic to supersonic and the species distribution varied from non-uniform to uniform. They include two turbine cascade flows (one was subsonic and the other was transonic) and two scramjet supersonic internal flows.

\section{Sample 1: High-speed Subsonic Flows in the NASA E ${ }^{3}$ Nozzle}

The first sample tested the code's generalizability to deal with both mono-species and multi-species, in other word, to check whether it had the same accuracy as the original mono-specie code. The blade profile and the experiment data were taken from the NASA report[13].The flow was air with inlet Mach number 0.12 and exit Mach number 0.8 . Three cases were calculated, including one component air by mono-species code with the same numerical method, one component air by multi-species code and air-like mixture of $79 \% \mathrm{~N}_{2}$ and $21 \% \mathrm{O}_{2}$ by multi-species code.

The mono-specie code's result provided a reference for the other two for comparison. Figure1 showed that the three Mach number contours were nearly the same and the static pressure distribution on the blade surface almost coincided with excellent agreement with the experiment data. The mono-species and multi-species code obtained almost the same answer for one component air, which testified to the multi-species code's generalizability and accuracy. The air-like mixture simulation showed the multi-species code's ability to deal with real multi-species flows.

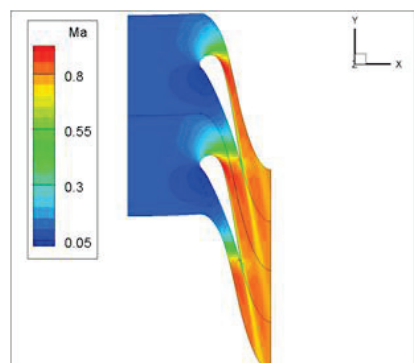

(a) one component air by mono-species code

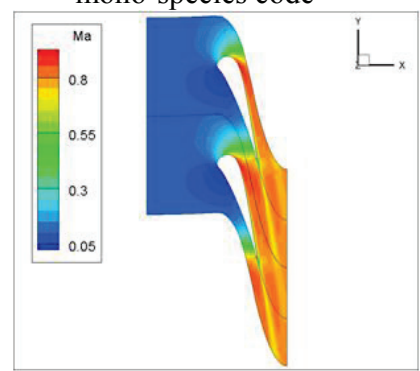

(c) $79 \% \quad \mathrm{~N}_{2}$ and $21 \% \mathrm{O}_{2}$ by multi-species code

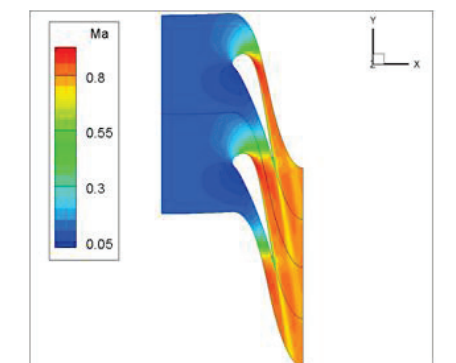

(b) one component air by multi-species code

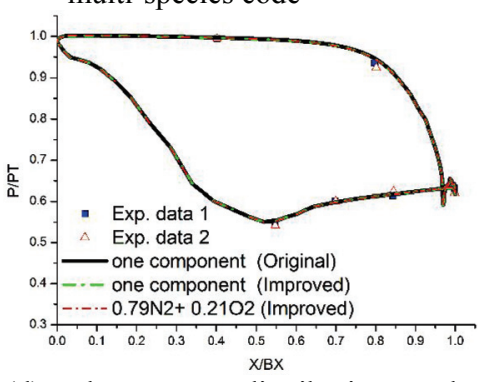

(d) The pressure distribution on the blade surface of the three cases
Figure 1 Mach number Contour and blade surface pressure distribution for sample 1

\section{Sample 2: Transonic Flows in the VKI 59 Cascade}

This second sample was a transonic flow problem. Its purpose was to test the code's ability to deal with the flow discontinuity, such as shock wave and material interface. The blade profile was from Von Karman Institution, and the experiment [14] was done at four famous wind tunnels in Europe. The transonic flow passed the blade channel with the inlet Mach number 0.2 and outlet Mach number 0.95 . The inlet flow composition was also air-like mixture with $79 \%$ Nitrogen and $21 \%$ Oxygen.

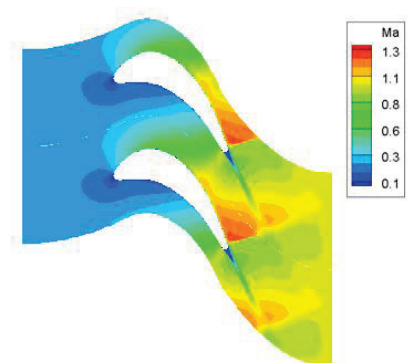

(a) Mach Number Contour at mid-span Figure 2

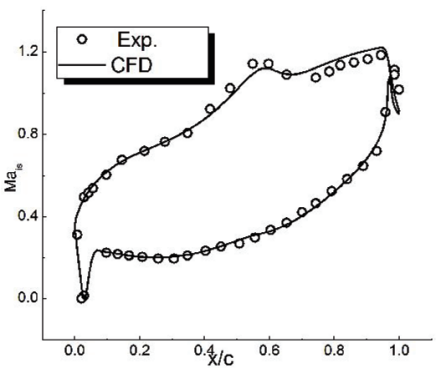

(b) Isentropic Mach number on the Blade Surface ach number contour and Blade surface Isentropic

The Mach number in Figure 2(a) showed that the flow accelerated over the suction side to sonic speed and then reduced to subsonic after a normal shock wave. The interaction between the wake 
and the shock wave was also captured by the multi-species code. Aside from these main flow features, the flow details like the slight compression wake emanating from the pressure side trailing edge, which was reflected by the suction side of the neighbour blade, was also captured in the simulation. The shock capture and detail flow reproduction highlighted the code's high resolution. What's more, the blade surface isentropic Ma also showed promising agreement with the experiment data.

\section{Sample 3: Supersonic Flows with Parallel Flow Ejection}

The third sample was a supersonic flow with species diffusion. Its role was to test the code's ability to deal with the supersonic flow and the accuracy to simulate the multi-species diffusion behaviour. The sample was taken from a model experiment of the scramjet engine, which was conducted by NASA[15]. The inlet flow was combustion gas consisting of $68 \%$ Nitrogen and $32 \%$ Oxygen with the Mach number 2.44. The Hydrogen was injected from a backward-facing step at sonic speed in a direction parallel to the main supersonic stream.

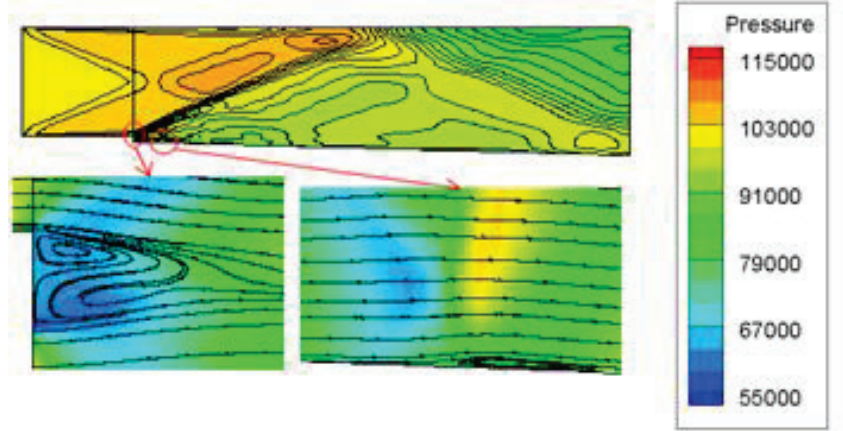

Figure 3 Pressure Distribution in the Flow Channel

The pressure field in Figure 3 showed that two oblique shock waves occurred at the inlet, intersecting with each other and reflecting on both the top and bottom walls. One rarefaction wave emanated from the backward-facing step and intersected with the reflected inlet oblique shock waves. The complex wave interaction in Figure 3 again testified to the code's resolution of the flow field. As to the flow field, the code captured many flow details, such as the backward recirculation and shock induced separation.

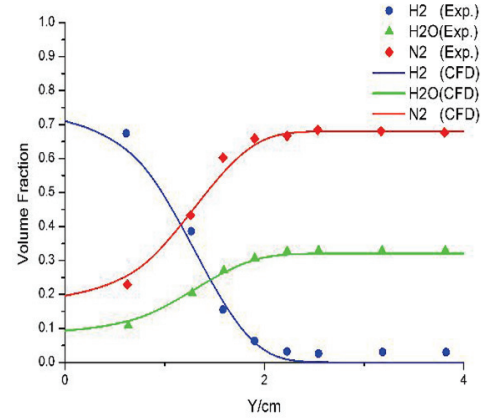

Figure 4 Species Mass Fraction Distribution at the Exit

As shown in Figure 4, the species mass fraction at the exit showed excellent agreement with the experiment data [15], which illustrated our code's accuracy for predicting species mixing.

\section{Sample 4: Supersonic Flow with Vertical Flow Ejection}

The fourth sample was the supersonic flow with transverse injection flow. It contained more flow physics so it is a more challenging task for code validation. This experiment was conducted by Kraemer and Rogers and reported by Weidner and Drummond [16].
The inlet flow was air with the Mach number 2.9 and the transverse flow was Helium injected from a slot at sonic speed.

As seen in Figure 5, the simulation illustrated complex flow physics in the transverse injection flow. The injection induced flow separation upstream which in turn induced an oblique shock wave. The sonic injection flow streamline formed a divergent nozzle, which accelerated the flow to supersonic and then reduced to a subsonic flow after a normal shock. Thus the Mach number distribution of the injection flow was like a disk, which was called Mach Disk. The Mach Disk interacted with the supersonic main flow forming a bow shock ahead of the injection flow. The injection flow reattached to the wall, which induced a recompression shock downstream. As to the streamline, the code not only captured the primary vortices induced by the injection, such as the upstream separation bubble and downstream recirculation, but also reproduced the secondary vortices induced by the primary ones.

The simulation was also compared with the experiment data quantitatively cross the flow direction downstream of the injection in Figure 6. The code's results agreed with the experiment data promisingly. However, the separation zone size upstream of the injection was underestimated by the turbulence model, which resulted in lower pressure peak in Figure 6(d).

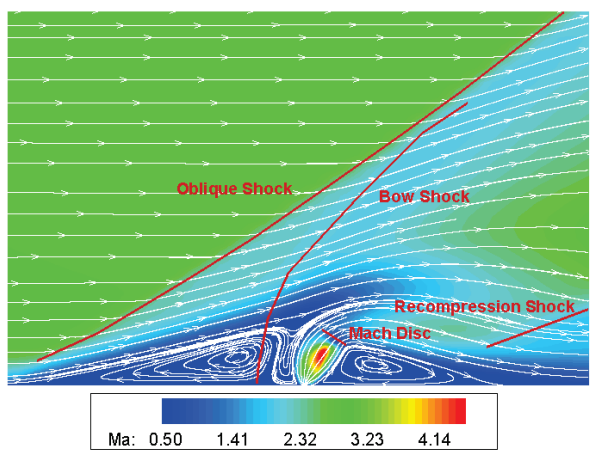

Figure 5 Mach number contour and streamline near the injection slot
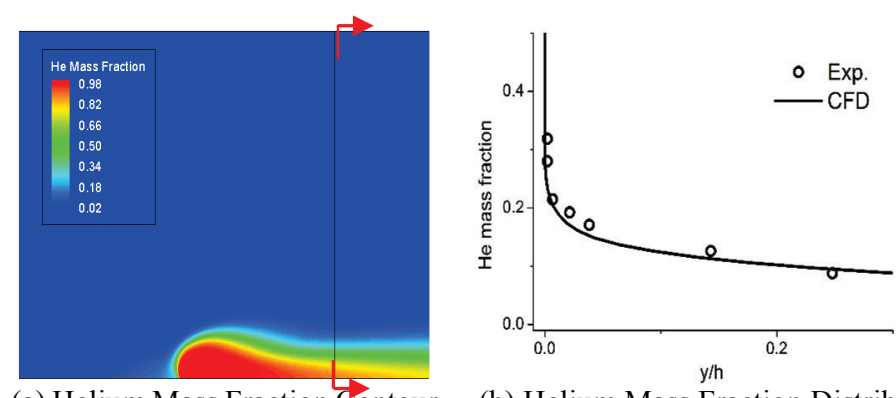

(a) Helium Mass Fraction Contour

(b) Helium Mass Fraction Distribu-

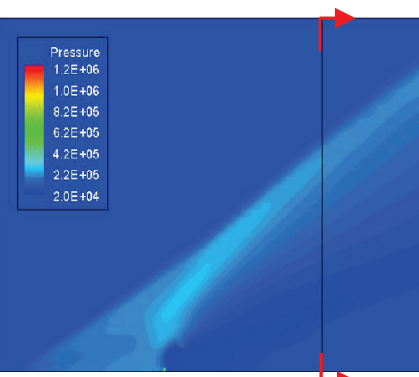

(c) Pressure Contour tion transverse the flow direction

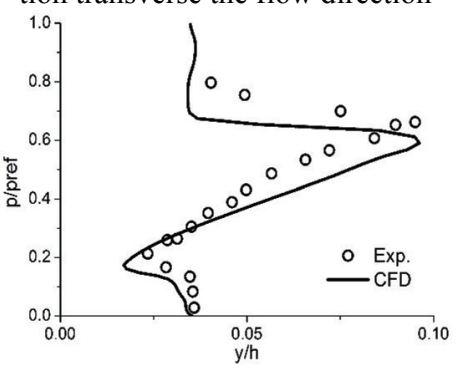

(d) Pressure Distribution transverse the flow
Figure 6 Mass Fraction and Pressure Distribution near the injection slot

The four typical validations above showed that the code had excellent flow field resolution and was accurate enough to species mixing. Then the code was employed to analyze the multi-species 
flow in a 1.5 -stage turbine.

\section{MULTI-SPECIES FLOWS IN A 1.5-STAGE TURBINE}

After the four validations for the numerical methods, great confidence was obtained to study a more complex flow problem, the unsteady multi-species flow in a 1.5-stage turbine. The experiment was conducted by R.J. Roback and R.P. Dring on the Large Scale Rotating Rig at UTRC[17]. The turbine rig consisted of 22 stators, 28 rotors at the first stage and 28 stators at the second stage, in which the axial spacing between the first stage stator and rotor was $15 \%$ of the average of their axial chords. The Q3D unsteady computation, to save computation consumption, was performed with special modelling in our 3D code. The blade number was scaled to $21: 28: 28$, with $3: 4: 4$ selected for computational domain.

\section{Cooling Effectiveness}

$\mathrm{CO}_{2}$, which traced the cooling flow and had the coolant-to-free stream density ratio of 1.5 , was ejected into the main flow at the trailing edge of the first stator. The accumulation of the coolant on the rotor was directly related to the local concentration of $\mathrm{CO}_{2}$, which implied adiabatic recovery temperature. When the coolant -to-free stream velocity ratio was 0.91 , the time averaged computed $\mathrm{CO}_{2}$ fraction distribution on the rotor was compared with experiment data. The $\mathrm{CO}_{2}$ fraction and surface distance were normalized in the same way as the experiment[18]. The surface distance was measured between the tangent points on the tangent line connecting the leading edge circle and trailing edge circle. The distance was defined as increasing positive along the suction side and negative along the pressure side.

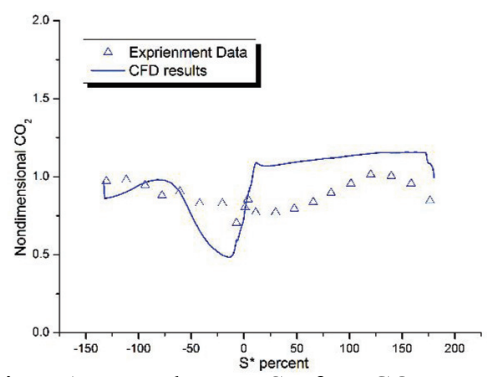

Figure 7 Time-Averaged Rotor Surface $\mathrm{CO}_{2}$ Fraction Distribution at Velocity Ratio 1.5

The Figure 7 showed that the numerical results showed the qualitative agreement with the experiment data. The difference between them may be caused by the Q3D assumption and the way that data was extracted. Although not accurate enough, the computation results fit the overall feature of the experiment, which implies that the primary flow physics was reproduced. So it was suitable for qualitative study.

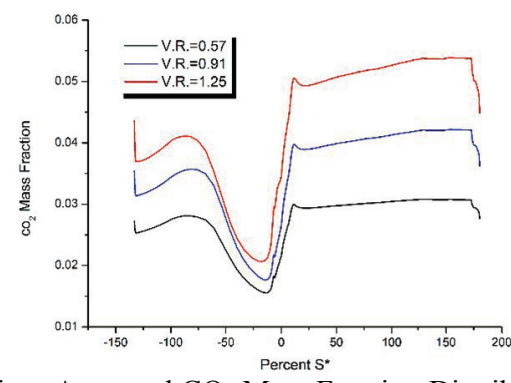

Figure 8 Time-Averaged $\mathrm{CO}_{2}$ Mass Fraction Distribution on the Rotor Surface for Three Velocity Ratios

Two other injection velocity ratios were investigated by unsteady simulation, while the flow coefficient $(\mathrm{Cx} / \mathrm{U})$ was kept at 0.78 . The results were time-averaged over one periodicity. The rotor surface $\mathrm{CO}_{2}$ distribution in Figure 8 showed larger amounts of coolant

flows accumulated on the rotor surface, as the velocity ratio increased. Meanwhile, the surface coolant distribution tended to be more non-uniform. These illustrated that the increased velocity ratio, which served as a double-edge sword, benefitted the rotor cooling but increased the temperature gradient of the rotor, which in turn increased the thermal stress inside the rotor. To give more insight into the non-uniformity, the time averaged $\mathrm{CO}_{2}$ mass fraction field in Figure 9 illustrated that the coolant had lower momentum than the main stream, so more coolant accumulated in the passage downstream of the stagnation point without reaching it. The high pressure resistance kept coolant coverage insensitive to the coolant velocity ratio changes.

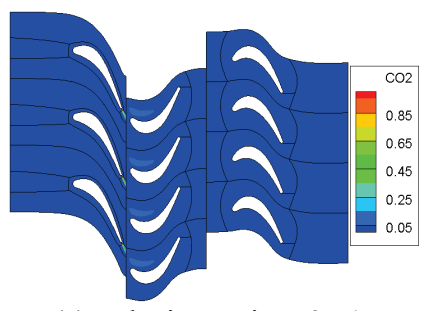

(a) Velocity Ratio $=0.51$

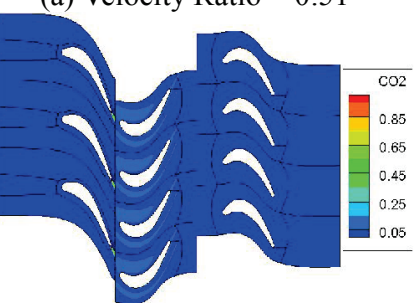

(c) Velocity Ratio $=1.25$

Figure 9 Time-Mean $\mathrm{CO}_{2}$ Mass Fraction Field for Three Velocity Ratios

\section{Loss Accounting}

In addition to the cooling effect, the loss induced by the trailing edge ejection was evaluated from the aerodynamic perspective. Following S. Friedrich, H.P. Hodson and W.N. Dawes[19], the mixed-out stagnation and static pressures were used together with a reference inlet stagnation pressure to determine loss. The loss coefficient $Y$ is defined as

$$
Y=\frac{P_{0 \text { ref }}-P_{0 \text { mixed }}}{P_{0 \text { ref }}-P_{\text {mixed }}}
$$

Different definitions of the inlet stagnation pressures will present different losses. Here we only wanted to discuss the losses caused by the injection in the external flow field not inside the cooling slot. Therefore, we chose the mass flow averaged stagnation pressure at stage inlet and coolant inlet as the inlet stagnation pressure. The mixed static and stagnation pressure was taken just downstream of the first stage stator by averaging with mass flow.

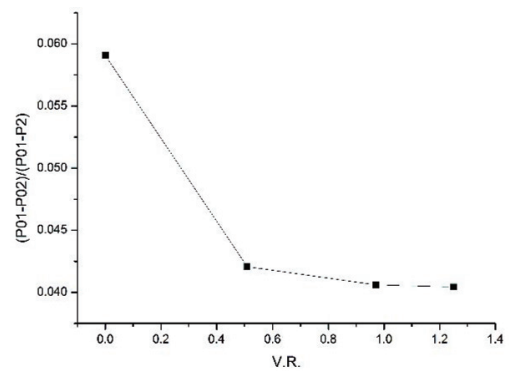

Figure 10 Loss Coefficient of Different Coolant-to-Free Stream Velocity Ratios

The Loss caused by the trailing edge injection can be decomposited into two categories, which was caused by two mechanisms. 
Firstly, the injected coolant mixed with the main stream induced mixing losses, which has negative effect on the aerodynamics of the turbine; Secondly, the trailing edge loss, which was elaborately discussed by Denton[20], was decreased by the injection coolant, which has positive effects on aerodynamics. The two mechanisms struggled with each other, which determined the overall aerodynamic loss. The Loss Coefficient for no injection and three velocity ratios $0.57,0.91,1.25$ in Figure 10 showed that the case with the trailing edge injection had lower losses than that without the injection and the losses decreased with the injection velocity ratio for the given range. It revealed that the second mechanism to reduce the trailing edge loss overtook the first one and the mixing process became more obvious, which was illustrated by the decreasing slope, as the velocity ratio increased.

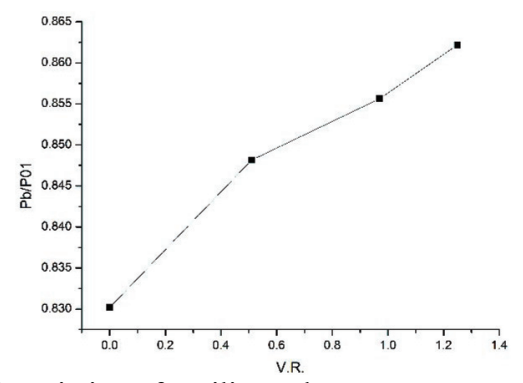

Figure 11 Variation of Trailing Edge Base Pressure with the Coolant Velocity Ratio

The mechanism can be further investigated. The trailing edge losses, analyzed by Denton[20], are caused by upstream boundary loss, the blockage caused by trailing edge thickness and trailing edge flow separation and wake mixing caused by low base pressure. Any method that affected these three factors will have influence on the losses. As seen in Figure 11, the base pressure was increased by the coolant injection, which in turn reduced wake mixing losses. The flow field in Figure 12 showed that the increasing coolant flow injection reduced the blockage by flow separation and mixing, which also contributed to the improvement of aerodynamic efficiency.

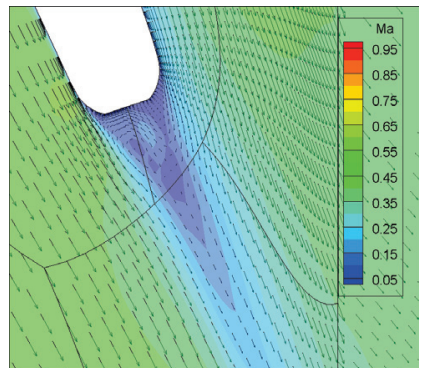

(a) no coolant injection

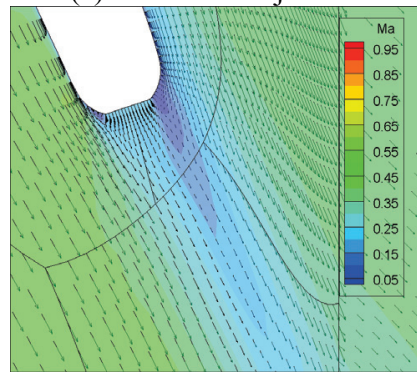

(c) V.R. $=0.91$

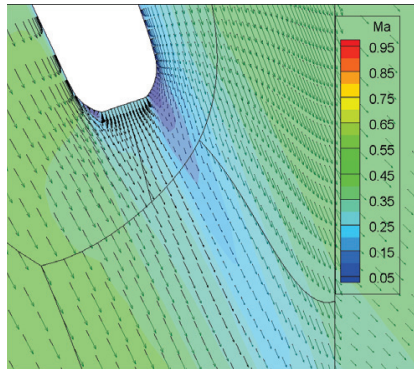

(b) V.R. $=0.57$

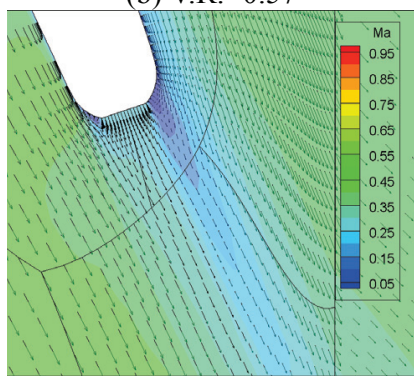

(d) V.R. $=1.25$
Figure 12 Trailing Edge Flow Field for Different Coolant-to-Free Stream Velocity Ratios

All in all, the trailing edge coolant injection has a large effect on both the cooling effect and the aerodynamic loss. As the coolant-to-free stream velocity ratio increases, the coolant is more likely to accumulate on the rotor surface non-uniformly and the aerodynamic losses at the trailing edge are reduced at a given range.

\section{CONCLUSION}

A high-fidelity numerical method for high-speed multi-species flow was developed to study the hot gases with different compositions in the turbine. Four validations, ranging from subsonic, transonic to supersonic, testified to its accuracy and resolution.

The multi-species flow in a 1.5-stage turbine, with coolant injection at the trailing edge, was investigated by the developed code. As the coolant-to-free stream velocity increases, the cooling effect on the downstream rotor improves. However, the temperature gradient is also increased, which in turn causes larger thermal stresses in the rotor. As to the aerodynamics, the coolant injection within a given range will improve the trailing edge flow efficiency by reducing the flow blockage and raising the base pressure.

In the future, it is necessary to simulate higher velocity ratio, in which the coolant flow speed exceeds the main flow at the stator exit, in order to study whether the characteristics of the cooling effectiveness and associated aero-losses at low velocity ratios will change.

\section{ACKNOWLEDGEMENTS}

This study was supported by Tsinghua-MHI R\&D Center, the National Natural Science Foundation of China (Grant No. 51136003) and the Special Funds for Major State Basic Research Projects (Grant No. 2007CB210108). Fruitful discussion with Dr. Xue-Li Gao from MHI is greatly acknowledged.

\section{REFERENCES}

[1] Martinez-Frias, J., Aceves, S.M., Smith, J.R., and Brandt,H., 2003, "A Coal-Fired Power Plant With Zero Atmospheric Emissions, Proceedings of AES-IMECE,2003 ASME International Mechanical Engineering Congress \& Exposition, IMECE2003-43923.

[2] Kizuka, N., Sagae, K., Anzai, S., Marushima, S., Ikeguchi, T., and Kawaike, K., 1999, "Conceptual Design of the Cooling System for 1700C-Class, Hydrogen-Fueled Combustion Gas Turbines," J. Eng. Gas Turbines and Power, 121, pp.108-115.

[3] Chiesa, P.,Lozza, G., and Mazzocchi, L., 2005, "Using Hydrogen as Gas Turbine Fuel," ASME Journal of Gas Turbines and Power, Vol. 127, No. 1, pp. 73-80.

[4] Mazzotta, D.W., Chyu, M.K., Karaivanov, V.G., Slaughter,W.S., and Alvin, M.A., 2008, "Gas-Side Heat Transfer in Syngas, Hydrogen-Fied and Oxifuel Turbines," ASME IGTI Paper GT2008-51474.

[5] Larrouturou, B., and Fezouri, L., 1989, "On the Equations of Multi-component Perfect of Real Gas Inviscid Flow", Lecture Notes in Mathematics, Volume 1402, pp.69-98.

[6] Poinsot, T., Veynante, D.,2005,"Theretical and Numerical Combustion(2nd edition)", R.T. Edwards Inc, Philadelphia, pp.13-15.

[7] McBride, B.J.,Zehe, M.J., and Gordon, S., 2002, "NASA Glenn Coefficients for Calculating Thermodynamic Properties of Individual Species", NASA/TP-2002-211556.

[8] Wilke, C.R., 1950, "A Viscosity Equation for Gas Mixtures", J. Chem. Phys., 18:517.

[9] Mason, E.A., and Saxena, S.C., 1958, "Approximate Formula for the Thermal Conductivity of Gas Mixtures", Phys. Fluids, $1: 361$.

[10] Kuo, K.K., 1986, "Principles of Combustion", John Wiley\&Sons, Inc.

[11] Yuan, X., and Daiguji H., 2001, "A special combined lower-upper factored implicit scheme for three-dimensional compressible Navier-Stokes equations", Journal of Computers Fluid, 2001, 30:339-363.

[12] Daiguji, H., Yuan, X., and Yamamoto, 1997, "Stabilization of higher-order high-resolution schemes for compressible Navier-Stokes equation", Int J Numer Methods Heat Fluid Flow, 7: 
250-274.

[13] Kopper, F.C., Milano, R., Davis, R.L. Dring, R.P., and Stoeffler, R.C., 1981, "Energy Efficient Engine High-Pressure Turbine Supersonic Cascade Technology Report", NASA-20646.

[14] Koiock, R., et al, 1986, "Transonic flow though a plane turbine cascade as measured in four European wind tunnels". ASME J Engng for Gas Turbines and Power, 108:277-84.

[15] Marshall, C., et. al, 1973, "Analytical and Experimental study of supersonic combustion of hydrogen in a vitiation airstream", NASA TM X-2828,1973

[16] Weidner, E., and Drummond, J., 1982, "Numerical Study of Staged Fuel-Injection for Supersonic Combustion", AIAA Journal, pp. 1426-1431.

[17] Dring, R.P., et. al, 1987, "Effects of Inlet Turbulence and Rotor/Stator Interactions on the Aerodynamics and Heat Transfer of a Large-Scale Rotating Turbine Model", NASA-23717

[18] Roback, R., Dring R., 1992, "Hot Streaks and Phantom Cooling in a Turbine Rotor Passage Part 1 - Separate Effects", ASME, 92-GT-75.

[19] Friedrichs, S., Hodson, H.P., Dawes, W.N., 1997, "Aerodynamic Aspects of Endwall Film Cooling", Journal of Turbomachinery", Vol.119, pp.786-793.

[20] Denton, J.D., 1993, "Loss Mechanisms in Turbomachines", Journal of Turbomachinery, Vol.115, pp.621-656. 